POLYMORPHONUCLEAR LEUKOCYTE (PMN) IN HEALTHY CHILDREN AFTER A SINGLE ORAL DOSE OF ERYTROMYCIN (E)

BARDARE M.,Viola E.,Biolchini A.,Clerici Schoeller M., Plebani A., Maccarinelli G.,Fraschini F.* Department of Pediatric Clinic of Milan University - via Commenda 9 20122 Milano - Italy

* Department of Chemotherapy of Milan University

The PMN, thanks to its ability to engulf and kill microorganisms, works as first defence against invading bacteria, whether or not antibiotics are administered. Because of the so-called "postantibiotic effect", antibiotics in vitro slow down bacterial growth and metabolism for a period stretching beyond the exposure.0ur study aims at assaying the capacity of $E$ to influence $P M N$ activity against microorganisms. PMN function was evaluated by chemotaxis, using the modified Boyden technique, and by Candida Albicans phagocytosis and killing using Lehre and Cline technique.PMN's were isolated from the peripheral blood of healthy children(mean age $7,16 \pm 2,82$ ) treated with a single oral dose of $25 \mathrm{mg} / \mathrm{Kg}$ of $\mathrm{E}$. Blood wase drawn prior to the administration of the antibiotic(reference value of PMN activity), 2 hrs. later (when E blood levels reach their peak) and $8 \mathrm{hrs}$. later( $2 \mathrm{hrs}$. after disappearance of $E$ from the blood). The results show that,from the 8 th hour on,there is a considerable increase in PMN phagocytic and killer activity (phF 0,17 ! $\pm 0,04$ before; $0,40 \pm 0,08$ after $8 \mathrm{hrs}$.; p $<0,001-\%$ killing $23,08 \pm 4,16$ before;32,6+5,1 after $8 \mathrm{hrs} . ; p<0,001$ ). Chemotaxis is unchanged (I.C. $78,52+7,73$ before; $77,72+8,28$ after $8 \mathrm{hrs}$.).

\section{WGPACI-Abstracts Read by Title}

\section{BRONCHIAL. PROVOCATION TESTS (BPT) WITH D.PTERONYSSINUS (D.PTEr). EFFECT OF SPECIFIC IMUUNOTHERAPY (SI).}

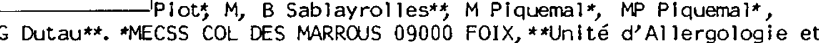
de Pneumologie infantile CHU RANGUEIL TOULOUSE

BPT with D.Pter were carried out on 29 asthmatic children, 7 to 15 years old, staying in a medical center in altitude. Each child presenled allergy to D.Pter. Tweive children received SI with D.Pter, and 3PT were performed before and after treatment.

Aerosol was delivered during normal tidal breathing from a nebulize of PIGLOWSKI (Ets Gauthier). Sal Ine, as control, was nebulized on the First day, and then a daily aerosol of D. Pter Alyostal Stallergene was Jelivered according to the fol lowing progression: 0.5IR, 1IR, 2IR, 4IR. Zesponse was monitored by flow-volume curves before aerosol, and $30 \mathrm{~min}$ Response was monitored by flow-volume curves before aerosol, and
$2 \mathrm{~h}$, $4 \mathrm{~h}$, and $9 \mathrm{~h}$ after it. The test was considered positive after a $2 \mathrm{~h}, 4 \mathrm{~h}$, and $9 \mathrm{~h}$ after it. The test was considered posit lve after a
fall of $20 \%$ of FEV1 and/or $30 \%$ of FEF50, compared with the value after saline.

No important asthma attack occured. The table shows the maximal dos of D.Pter ( $X \cdot D P$ ) and the time of reaction after aerosol: Irmediate at $30 \mathrm{~min}$ alone (I), delayed at $9 \mathrm{~h}$ alone (D), immediate and delayed (ID), absent $(A)$ before and after SI:

\begin{tabular}{|c|c|c|c|c|c|c|c|c|}
\hline & & X DP & & ] & D & ID & A & $n$ \\
\hline before SI & $\bar{x}=1$ & $\sigma=0.77$ & $\mathrm{~S}$ & 2 & 4 & 6 & - & 12 \\
\hline after SI & $x=2.31$ & $\sigma=1.21\}$ & $p<0.05$ & 6 & 3 & 2 & 1 & 12 \\
\hline
\end{tabular}

Delayed react lons are very frequent $(10 / 12)$ before SI. SI provokes an increase of X DP with significant difference and a decrease of the number of delayed responses $(5 / 12)$

\section{4}

CHRONIC URTICARIA IN PEDIATRIC PATIENTS Department of Pediatric Clinic of Milan Univeristy - via Commenda 9 - 20122 Milano - Italy -

The pathogenesis of chronic urticaria is still an unsolved problem. Aim of this study is to evaluate the incidence of food allergy and food pseudoallergy in a pediatric population. In 97 children suffering from chronic urticaria starting between 1 mo. and $12 \mathrm{yrs}$. the following tests were carried out:common blood chemistry, specific and total IgE radioimmunoassay,prick-tests with the most common allergens, exclusion diet followed by challenge. In a group of patients with suspected pseudoallergy challenge with Sodium Benzoate, Aspirin and Tartrazine was carried out.Blood chemistry always assayed normal. There was evidence of food intolerance in 61 children ( 14 IgE-mediated and 47 non IgEmediated). In other 24 cases food intolerance was undoubtly ruled out while in the remaining 12 cases urticaria disappeared on elimination diet but did not recur on challenge. 15 of the 24 cases with no food intolerance were diagnosed as cholinergic urticaria ( 5 cases), infectious urticaria ( 2 cases), inhalant urticaria ( 8 cases); for the remaining 9 patients it was concluded for idiopathic urticaria. The Idditives challenge yelded positive results in almost $20 \%$ of cases.
SOYBEAN INTOLERANCE IN CHILDREN WITH FOOD ALLERGY

BARDARE M., Zani G., Folchi vici E., Magnolfi C., Varin E Deprtment of Pediatric Clinic of Milan University Via Commenda 9 20122 Milano- Italy

The use of soy milk as an alternative formula in cow milk intolerance and its role in inducing soy seed hypersensitivity is still controversial. In our study soybean intolerance was evaluated in 71 children ( age range 8 mo - 15 yrs) affected with IgE-mediated ( 50 subjects) and non-IgE-mediated ( 21 subjects) food intolerance (urticaria, atopic dermatitis, rhinitis and asthma) and a rlationship between soy milk, cow milk and seed intolerance was assessed. Soy bean specific IgE assayed positive in $46 \%$ of children with food allergy and negative in all cases of non-IgE-mediated food intolerance. The soy bean hypersensitivity was not related to a prior soy milk feeding. Soy RAST was in agreement with seed RAST in $82 \%$ of cases, with cow milk RAST in only $54 \%$ of cases. Intolerance signs and symptoms appeared at soy bean challenge in $18 \%$ of patients: the incidence was not related to a preceding soy milk feeding.

There was a relationship between soy milk and cow milk intolerance in $23 \%$ of cases, between soy milk and seed intolerance in $41 \%$ of cases.

\section{ASTHMA AND ENVIRONMENTAL FACTORS}

Department of Pediatrics, Hospital Nuestra

Señora del Mar, Autonomous University of Barcelona, Barcelona, Spain

We evaluated the clinical data of 893 children with asthmatic crisis treated in the emergency room at our institution between January 1, 1983 and December 31 , 1984. Age and sex of the patients as well as the number of cases admitted to the hospital in relation to the total number of patients that consulted the emergency service were analyzed, and a correlation with environmental conditions (air pollution, meteorological data, pollen levels) was established. The highest incidence of bronchospasms was observed in the fall, but no significant relationship was noticed between but no significant relationship was noticed between tration of airborne pollutants. During high-pollution days, however, there was an increase in the number of asthmatic attacks. Analysis of variance showed a significant correlation between asthmatic crises and air pollution in children under 3 years of age. These results should be cautiously considered and only results should be cautiously considered and only
further studies over the next years can provide definite conclusions.

\section{TERM REMISSION.}

\section{G.Longo,F.Poli,R.Strinati}

University paediatrics Clinic, Istituto per l'Infanzia, Trieste, Italy It is not known if asthmatics represent a separate hyperreactive population from the normal distribution of afrways reactivity (AR), or if they merely represent a hyperreactive extreme of a normal distribution. In a recent study about AR (Lancet $i 1,792$, I983) our findings suggested the first hypothesis: this may imply that "once asthmatic, always an asthmatic". On these assumptions, we studied the bronchial reactivity to Carbacholine in I2 subjects with a remote hystory of wheezy bronchitis during childhood, and since then free of asthma or wheezing for, at least, IO years ( $\bar{x} 2 \mathrm{I} .2$ years). Stepped doses of Carbachol Cloride (0. $1 \%$ ) aerosol (Bronchotest. Dr.J.Piglowsky, Paris) were given until a $50 \%$ drop in forced expiratory flow at $25-75 \%$ was observed, or until the subject had inhaled 80 times his own forced vital capacity equivalent of the aerosol. All I2 patients were found to have $A R$ in the asthmatic range (data obteined in 50 asthmatics). The persistence of tranchial hyperreactivity during long term symptom free periods,implies that bronchial hyperreactivity may be a constitutional inherited character which may become clinically manifest if the subjects are exposed to exogenous factors such as environmental irritants (no one of our patients had an occupational risk), infectious agents,or allergens, even after subsidence of asthma symptoms for many years. The lowest values in the asthmatic range were observed in our patients with at least an allergy skin prick test positive la weal 3 $\mathrm{mm}$ or more in diameterlor smokers. It is possible that if these risk factors had been present in those subjects with greatly increased non specific AR, they would have been asthmatic. 